\title{
Situações Interpessoais Difíceis: Relações entre Habilidades Sociais e Coping na Adaptação Acadêmica
}

\author{
Adriana Benevides Soares ${ }^{1}$ \\ ${ }^{1}$ Universidade Salgado de Oliveira, SP, Brasil. \\ Marisangela Siqueira de Souza ${ }^{1}$ \\ ${ }^{1}$ Universidade Salgado de Oliveira, SP, Brasil. \\ Humberto Cláudio Passeri Medeiros ${ }^{1}$ \\ ${ }^{1}$ Universidade Salgado de Oliveira, SP, Brasil.
}

\author{
Marcia Cristina Monteiro ${ }^{1}$ \\ ${ }^{1}$ Universidade Salgado de Oliveira, SP, Brasil. \\ Fátima Almeida Maia ${ }^{1}$ \\ ${ }^{1}$ Universidade Salgado de Oliveira, SP, Brasil. \\ Roberta de Souza Nogueira Barros ${ }^{1}$ \\ ${ }^{1}$ Universidade Salgado de Oliveira, SP, Brasil.
}

Resumo: Este estudo teve como objetivo verificar as relações preditivas entre as variáveis habilidades sociais e estratégias de copingna adaptação à universidade em situações consideradas de difícil manejo no contexto universitário. As cinco situações elencadas foram: "Pedir a um colega que desligue seu celular na aula", "Apresentar trabalho em aula", "Pedir aos colegas que evitem de lanchar na sala", "Mobilizar colegas para reivindicar direitos", "Receber críticas do professor". Participaram da pesquisa 400 universitários, sendo 61,5\% mulheres $(\mathrm{N}=246)$ com idades entre 18 e 60 anos $(\mathrm{M}=25,78$ e DP = 7,99). Foram utilizados os seguintes instrumentos: Inventário de Habilidades Sociais, Questionário de Vivências Acadêmicas reduzido (QVA-r) e a Escala Modos de Enfrentamento de Problemas (EMEP). Os resultados demonstraram que o estudante que recorre às estratégias de enfrentamento Focalizado no Problema e na Busca de Suporte Social lida melhor com questões de difícil manejo na universidade. A estratégia de enfrentamento Focalizado no Problema e a Habilidade Social de Autoafirmação na Expressão de Sentimento Positivo apresentaram maior impacto nos escores de adaptação acadêmica. Os resultados contribuem para ampliar o entendimento sobre o uso dos processos cognitivos e comportamentais associados à adaptação ao Ensino Superior, o que possibilita a criação de recursos, por parte das universidades, para dar suporte aos estudantes nesse processo.

Palavras-chave: Adaptação Acadêmica, Habilidades Sociais, Estratégias de Coping.

\section{Difficult Interpersonal Situations: Relations between Social Skills and Coping in Academic Adaptation}

\begin{abstract}
This study aimed to verify the predictive relationships between the variables social skills and coping strategies in adaptation to the university in situations considered difficult to manage in the university context. The five situations listed were: "Ask classmates to turn off their cell phone in class", "Present class work", "Ask classmates to avoid lunch in the classroom", "Mobilize classmates to claim rights", "Receive teacher criticism". 400 university students participated in this study, $61.5 \%$ women $(\mathrm{N}=246)$, aged between 18 and 60 years $(\mathrm{M}=25.78$ and $\mathrm{SD}=7.99)$. The following instruments were used: Social Skills Inventory, Reduced Academic Experience Questionnaire and Modes of Confronting Problems Scale. The results showed that the student who uses strategies of coping focused on the problem and on the search for social support better deals with issues of difficult management at university. The coping strategy focused on the problem and the social skill of self-affirmation in the expression of positive feeling presented greater impact in the academic adjustment scores. The results contribute to a better understanding of the use of cognitive and behavioral processes associated with adaptation to Higher Education, which allows the creation of resources by the universities to support students in this process.
\end{abstract}

Keywords: Academic Adaptation, Social Skills, Coping Strategies. 


\title{
Situaciones Interpersonales Difíciles: Relaciones entre Habilidades Sociales y Coping en la Adaptación Académica
}

\begin{abstract}
Resumen: Este estudio tuvo como objetivo verificar las relaciones predictivas entre las variables habilidades sociales y estrategias de coping en la adaptación a la universidad en situaciones consideradas de difícil manejo en el contexto universitario. Las cinco situaciones relacionadas fueron: "Pedir a un colega que apague su teléfono en la clase", "Presentar trabajo en clase", "Pedir a los colegas que eviten de comer en la sala", "Movilizar a los colegas para reclamar derechos", "Recibir críticas del profesor". En la investigación participaron 400 universitarios, siendo $61,5 \%$ mujeres $(\mathrm{N}=246)$ con edades entre 18 y 60 años $(\mathrm{M}=25,78$ y $\mathrm{DP}=7,99)$. Se utilizaron los siguientes instrumentos: Inventario de Habilidades Sociales, Cuestionario de Vivencias Académicas reducido (QVA-r) y la Escala Modos de Enfrentamiento de Problemas (EMEP). Los resultados demostraron que el estudiante que recurre a las estrategias de enfrentamiento Focalizado en el Problema y en la Búsqueda de Soporte Social hace frente mejor con cuestiones de difícil manejo en la universidad. La estrategia de enfrentamiento enfocada en el problema y la Habilidad Social de Autoafirmación en la Expresión de Sentimiento Positivo, presentaron un mayor impacto en los escores de adaptación académica. Los resultados contribuyen a ampliar el entendimiento sobre el uso de los procesos cognitivos y comportamentales asociados a la adaptación a la Enseñanza Superior, lo que posibilita la creación de recursos, por parte de las universidades, para dar soporte a los estudiantes en este proceso.
\end{abstract}

Palabras clave: Adaptación Académica, Habilidades Sociales, Estrategias de Coping.

\section{Introdução}

A adaptação à universidade é uma etapa na vida estudantil que exige do aluno maior autonomia nos estudos, maturidade para gerir diferentes componentes curriculares, novas relações interpessoais e o compromisso com o processo de ensino e aprendizagem (Clinciu, 2012). A transição e adaptação acadêmicas são fenômenos complexos e vivenciados de forma diferente pelos estudantes (Almeida, Araújo, \& Martins, 2016). As demandas na transição do Ensino Médio para o Superior exigem do aluno altos níveis de independência, iniciativa, autorregulação e estratégias de enfrentamento para gerir as adversidades (Fagundes, 2012). A construção de novas relações, o afastamento de familiares, o ajustamento a novas regras institucionais, como também as exigências pessoais, sociais e acadêmicas são algumas das demandas desse processo de transição (Manee, Khoiee, \& Eghbal, 2015; Santos, Oliveira, \& Dias, 2015).

Desta forma, principalmente no primeiro ano do curso universitário, o estudante depara-se com um ritmo novo de vida caracterizado por transformações que exigirão habilidades para adequar-se às novas contingências do ambiente universitário, o que pode gerar estresse (Assis, Silva, Lopes, Silva, \& Santini, 2013; Clinciu, 2012; Feldt, Graham, \& Dew, 2011; Manee et al., 2015). O universitário, ao ser confrontado com eventos ameaçadores, pode reagir utilizando estratégias de enfrentamento (coping) - que se referem aos recursos emocionais, cognitivos e comportamentais utilizados pelos indivíduos na tentativa de lidar com situações estressoras (Baker, 2003; Costa, \& Leal, 2006; Lazarus, \& Folkman, 1984). O coping é um processo regulatório que pode reduzir os sentimentos negativos gerados por situações estressantes. Em sentido mais estrito, o termo coping tem sido usado como uma resposta necessária e importante na adaptação do estudante ao contexto acadêmico (Crockett et al., 2007; O'Brien, Mathieson, Leafman, \& Rice-Spearman, 2012; Santeiro, Yoshida, Peixoto, Rocha, \& Zanini, 2016).

As estratégias de enfrentamento utilizadas pelos discentes para reagirem a momentos de mudança e em situações estressoras têm sido tema de investigação com estudantes universitários. (Carelli, \& Santos, 1998; Clinciu, 2012; González-Torres, \& Artuch-Garde, 2014; Manee et al., 2015; Mofidi, El-Alayli, \& Brown, 2014). Segundo Seidl, Tróccoli e Zannon (2001), as 
estratégias de coping podem ser classificadas como Focalizadas no Problema, na Emoção, no Suporte Social e em Práticas Religiosas/Pensamento Fantasioso. O primeiro é compreendido como um esforço em solucionar ou modificar a situação que originou o estresse. O segundo, como o esforço cognitivo e comportamental para buscar soluções paliativas de enfrentamento ou afastamento do elemento estressor. O terceiro diz respeito ao apoio instrumental, informacional e emocional do outro e, o quarto, representa pensamentos e comportamentos religiosos para enfrentar o problema. Alguns estudos relatam a relação entre estratégias de enfrentamento e adaptação acadêmica (Carlotto, Teixeira, \& Dias, 2015; Crockett et al., 2007; Kuncharin, \& Mohamad, 2014; Oliveira, Carlotto, Vasconcellos, \& Dias, 2014; Sicre \& Casaro, 2014), mas nenhum foi encontrado que evidenciasse a relação entre coping e situações interpessoais específicas neste contexto tais como: "Pedir a um colega que desligue seu celular na aula", "Apresentar trabalho em aula", "Pedir aos colegas que evitem de lanchar na sala", "Mobilizar colegas para reivindicar direitos" e "Receber críticas do professor". Entender como os estudantes reagem, se de forma mais passiva ou ativa em situações distintas, pode favorecer uma abordagem mais direcionada pelos centros de apoio aos estudantes objetivando uma melhor adaptação ao ambiente universitário, buscando minimizar problemas como abandono e desistência.

Carlotto, Teixeira e Dias (2015) realizaram pesquisa sobre adaptação acadêmica e coping com 412 universitários. Concluíram que o coping Focalizado no Problema esteve mais relacionado com as dimensões Estudo, Institucional e Carreira do Questionário de Vivências Acadêmicas reduzido (QVA-r) - instrumento que permite investigar as vivências acadêmicas em cinco dimensões: Pessoal, associada às percepções de bem-estar por parte do estudante; Interpessoal, diz respeito ao relacionamento com pares e as competências necessárias para relações mais significativas; Vocacional, centrados na carreira, profissão e satisfação com o curso; Estudo-Aprendizagem, diz respeito as competências de estudo e situações do cotidiano de trabalho acadêmico e Institucional, aborda o interesse geral pela instituição. As estratégias Focadas na Emoção estiveram correlacionadas negativamente com a adaptação acadêmica, especificamente, na dimensão que trata do ajustamento pessoal. A estratégia base- ada no Suporte Social correlacionou-se de forma positiva com a dimensão Interpessoal. No entanto, observou-se que altos escores de Suporte Social estiveram associados com baixos escores na dimensão Pessoal do QVA-r, indicando que a procura de suporte pode sugerir uma inabilidade em lidar com os estressores de forma individual. Finalmente, as correlações entre as Práticas Religiosas/Pensamento Fantasioso associaram-se de forma negativa à dimensão Pessoal.

Em estudo bibliométrico, Oliveira, Carlotto, Vasconcellos e Dias (2014) identificaram relações entre adaptação acadêmica e estratégias de enfrentamento em universitários. A análise de 12 trabalhos indicou que as estratégias de coping evitativas podem estar associadas a níveis inferiores de adaptação acadêmica, enquanto as baseadas no confronto, na resolução de problemas, na flexibilidade, no humor e no suporte social têm maiores níveis adaptativos.

A adaptação à universidade requer, além de estratégias de enfrentamento, habilidades sociais que permitam que o estudante faça novas amizades, se relacione com professores, gestores e funcionários de maneira adequada atingindo seus objetivos e contribuindo para uma melhor saúde física e mental. A. Del Prette e Del Prette (2003) reforçam que as habilidades interpessoais são importantes para a liderança de equipe, a administração do estresse, a resolução de problemas e a tomada de decisões, a criatividade, assim como para a destreza de falar em público. As habilidades sociais podem ser entendidas como classes de comportamentos de um indivíduo requeridas em seu convívio, que contribuem para um desempenho socialmente competente (Del Prette, \& Del Prette, 2001, 2010).

Alguns estudos evidenciaram a relação entre habilidades sociais e adaptação à universidade. Manee et al. (2015) realizaram uma intervenção com 100 alunos de primeiro período de uma universidade no Irã e encontraram que as habilidades sociais têm efeito positivo significativo na adaptação acadêmica. Com 99 estudantes Gerk e Cunha (2006) identificaram maiores valores de correlação entre o escore total do Inventário de Habilidades Sociais (IHS) e as subescalas do QVA: Relacionamento com os Colegas, Autonomia Pessoal, Autoconfiança e Percepção Pessoal de Competências Cognitivas.

Soares, Poubel e Mello (2009) realizaram um estudo comparativo em instituições de ensino 
público e privado na cidade do Rio de Janeiro com 200 estudantes do curso de Psicologia. Foi evidenciado que, nas instituições públicas, um bom repertório de habilidades sociais estava correlacionado com as dimensões de vivências acadêmicas Pessoal e Contextual, nas particulares, um bom repertório de habilidades sociais se correlacionava com a dimensão de Realização. Concluiu-se que, na universidade pública, em que a exigência para o acesso era maior, os alunos confirmavam suas expectativas de destreza intelectual e tinham apoio das famílias. Os estudantes das universidades privadas visavam o mercado de trabalho para um retorno mais rápido do investimento feito com o curso. A esse respeito, Iksan et al. (2012) destacam a necessidade de as habilidades sociais fazerem parte do currículo acadêmico. Sendo a universidade pública ou privada, tais habilidades serão essenciais durante todo processo de formação e para o desenvolvimento futuro da carreira desses estudantes.

Em estudo com 500 alunos, Soares, Mello e Baldez (2011) encontraram correlações de todas as dimensões das vivências acadêmicas com os fatores de habilidades sociais para estudantes da rede pública, enquanto que, para a rede privada, encontraram menos associações principalmente na dimensão Pessoal e nas habilidades Assertivas. Em estudo mais recente, Soares, Francischeto, Peçanha, Miranda e Dutra (2013), com uma amostra de 393 estudantes, verificaram que todas as dimensões de vivências acadêmicas se correlacionavam com o escore total de habilidades sociais e que o escore de vivências acadêmicas se correlacionava com todos os fatores de habilidades sociais.

Soares, Mourão, Santos e Mello (2015) caracterizaram os universitários com relação ao seu repertório de habilidades sociais e as vivências acadêmicas, apresentando a correlação entre as variáveis em 202 alunos. Apontaram escores médios tanto da vivência acadêmica quanto do repertório de habilidades sociais e correlações significativas fracas entre essas variáveis.

Soares, Seabra e Gomes (2016a), em pesquisa com 689 universitários do primeiro ano, indicaram que os alunos de instituições particulares apresentam melhores percepções das vivências acadêmicas e das habilidades sociais do que das públicas. Também encontrou que os estudantes da metrópole são melhores adaptados à universidade e têm mais habili- dades sociais do que os do interior, evidenciando que pertencer às instituições privadas e a grandes cidades favorece a adaptação acadêmica dos graduandos.

A adaptação acadêmica é um processo complexo e multideterminado (Albanaes, Bardagi, Luca, \& Girelli, 2014; Bisinoto, Rabelo, Marinho-Araújo, \& Fleith, 2016), pois envolve diferentes aspectos que apresentam associação entre si (Manee et al., 2015; Mofidi et al., 2014; Soares et al., 2016a), como os referentes ao aluno (cumprimento de cronogramas e a organização pedagógica para estudar); institucionais (investimento em laboratórios e bibliotecas) e sociais (atividades que possibilitem a troca com pares). Entretanto, muito se avança nesta temática a partir de investigações que abarcam modelos causais de análise, modelos que apontem que a variável de critério tem associação com variáveis preditoras (Pasquali, 2015). A compreensão mais detalhada da reação dos discentes para cada situação estressora pode favorecer intervenções mais específicas e efetivas para minimizar as dificuldades enfrentadas. Tendo em vista os estudos arrolados, não foi encontrado nenhum que apresentasse associação entre os três construtos em tela. Além disso, a literatura apresenta resultados de estudos correlacionais, tanto relativos a estratégias de enfrentamento quanto as habilidades sociais e adaptação acadêmica, porém não são estudadas situações específicas de estresse nas relações interpessoais que possam dificultar a adaptação à universidade (Soares, \& Del Prette, 2013; Soares et al., 2016a). Portanto, o objetivo desse estudo foi o de verificar: 1) se existem relações entre as situações interpessoais e as formas de enfrentamento realizado (de forma geral e de forma específica a cada situação estudada) e 2) se as variáveis habilidades sociais e coping são preditoras da adaptação à universidade.

\section{Método}

\section{Participantes}

Participaram 400 estudantes universitários de cursos e períodos diversos, sendo que $34,5 \%(\mathrm{~N}=138)$ eram alunos do curso de Psicologia. Na amostra, $61,5 \%$ eram mulheres $(\mathrm{N}=246)$ com idades que variavam de 18 a 60 anos $(M=25,78$ e $\mathrm{DP}=7,99)$. Eram solteiros $80,5 \%(\mathrm{~N}=322)$, pertenciam a uma instituição privada $82,5 \%(\mathrm{~N}=330)$. De classes sociais variadas com predominância da classe B2 43\% (N = 172). 


\section{Instrumentos}

O Inventário de Habilidades Sociais (IHS) (Z. Del Prette \& Del Prette, 2001) é constituído de 38 itens. Avalia cinco fatores: 1 - Enfrentamento e Autoafirmação com Risco - (11 itens) remete a situações interpessoais que levam o respondente a reagir pela afirmação e defesa de direitos e autoestima, com risco potencial de reação indesejável - $(\alpha=0,96) .2$ - Autoafirmação na Expressão de Afeto Positivo - (sete itens) aponta demandas interpessoais de expressão de afeto positivo e de afirmação de autoestima, possibilitando risco mínimo de situação indesejável $-(\alpha=0,86)$. 3 - Conversação e Desenvoltura Social - (sete itens) avalia a capacidade de lidar com situações sociais neutras, com um risco mínimo de reação indesejável pelo outro - $(\alpha=0,81) .4$ - Autoexposição a Desconhecidos ou a Situações Novas - (quatro itens) envolve pequeno risco de reação indesejável do interlocutor - $(\alpha=0,75) .5$ - Autocontrole da Agressividade - (três itens) remete a situações adversas com razoável controle da raiva e da agressividade - $(\alpha=0,74)$. Na análise de consistência interna foi obtido coeficiente Alpha de Cronbach de 0,75.

O Questionário de Vivências Acadêmicas - reduzido (QVA-r) (Almeida, Soares, \& Ferreira, 2002) é constituído por 55 itens (Granado, Santos, Almeida, Soares, \& Guisande, 2005). O instrumento permite investigar cinco dimensões: Pessoal, Interpessoal, Vocacional, Estudo-Aprendizagem e Institucional. A dimensão Pessoal (14 itens) está associada às percepções de bem-estar por parte do estudante e apresenta $\alpha=0,84$. A dimensão Interpessoal (12 itens) $(\alpha=0,82)$, diz respeito ao relacionamento com pares e às competências necessárias para relações mais significativas. A Vocacional (12 itens) $(\alpha=0,86)$ centrados na carreira, profissão e satisfação com o curso. A dimensão Estudo-Aprendizagem (9 itens) $(\alpha=0,78)$ e diz respeito as competências de estudo e situações do cotidiano de trabalho acadêmico. Finalmente, a Institucional $(\alpha=0,77)$ (oito itens) aborda o interesse geral pela instituição de Ensino Superior, incluindo o conhecimento e a apreciação dos serviços e a infraestrutura da instituição.

A Escala Modos de Enfrentamento de Problemas (EMEP) (Vitaliano, Russo, Carr, Maiuro, \& Becker, 1985, adaptado para o português por Gimenes \& Queiroz, 1997 e validada para a população brasileira por Seidl et al., 2001) é um instrumento de autorrelato que conta com 45 itens distribuídos entre 4 fato- res: Enfrentamento Focalizado no Problema, Enfrentamento Focalizado na Emoção, Busca de Prática Religiosa/Pensamento Fantasioso e Busca de Suporte Social que englobam pensamentos e ações que o indivíduo utiliza para lidar com as demandas externas e internas numa situação estressante. $\mathrm{O}$ fator Enfrentamento Focalizado no Problema (18 itens) refere a uma reação positiva de aproximação da situação estressora com intuito de solucioná-la $(\alpha=0,78)$. O fator Enfrentamento Focalizado na Emoção (15 itens) inclui reações emocionais negativas como raiva, culpa e tensão $(\alpha=0,83)$. O fator Busca de Prática Religiosa/Pensamento Fantasioso (sete itens) remete a um enfrentamento que recorre a sentimentos como fé e esperança ( $\alpha=0,64)$. Por fim, o fator Busca de Suporte Social (cinco itens) indica a procura por informações, conselhos e acolhimento para o enfrentamento do problema $(\alpha=0,61)$.

Cinco situações acadêmicas consideradas difíceis foram introduzidas no cabeçalho do EMEP. As situações correspondem à pesquisa anterior (Soares, Gomes, Maia, C. Gomes, Monteiro, 2016b) na qual os autores identificaram situações consideradas difíceis no contexto acadêmico. As situações foram inseridas de forma interrogativa. A primeira investiga como o aluno pensa e reage quando precisa pedir a um colega que desligue seu celular na aula porque se sente incomodado; a segunda questiona como lida quando precisa apresentar um trabalho em uma situação pública para uma audiência de estudantes e professores; a terceira, quando quer pedir aos colegas que evitem lanchar em aula porque se sente incomodado; a quarta, quando tem que mobilizar colegas para reivindicar direitos junto a autoridade pertinente e a quinta aborda como o aluno reage quando recebe críticas do seu professor pela apresentação do seu trabalho.

\section{Procedimentos}

Foram aplicados os questionários de forma coletiva em turmas variando de 20 a 40 alunos universitários na seguinte sequência: IHS, QVA-r, EMEP. Foram coletados dados sociodemográficos. Todos assinaram Termo de Consentimento Livre e Esclarecido. O presente estudo foi aprovado no Comitê de Ética da Universidade.

Para investigar quais eram as estratégias de enfrentamento mais utilizadas (de forma dependente e independente das situações interpessoais) 
foram computadas por meio do SPSS as variáveis de diferença para todos os pares das diferentes estratégias de enfrentamento. Por meio do teste $t$ de Student para uma amostra (One-sample t-test) foi avaliado se a média dos escores dessa variável era estatisticamente similar a zero, o que atestaria igualdade na obtenção dos escores das duas estratégias. Por fim, buscou-se investigar o impacto das variáveis estratégias de enfrentamento e habilidades sociais na adaptação acadêmica. Foram implementadas análises de regressão linear múltipla (RLM) (método Enter) para avaliar o impacto das VIs (estratégias de enfrentamento e habilidades sociais) na VD (adaptação à universidade).

\section{Resultados}

Para os resultados relativos a comparação dos escores das estratégias de enfrentamento independente das situações, todos os fatores se diferenciaram entre si: Focalizado no Problema/Emoção $(t=15,18$; $p<0,00)$; Problema/Fantasioso $(t=5,40 ; p<0,00)$; Problema/Suporte Social $(t=1,56 ; p<0,120)$; Emoção/Fantasioso $(t=-8,87 ; p<0,00)$; Emoção/Suporte Social $(t=-13,85 ; p<0,04)$; Fantasioso/Suporte Social $(t=-4,07 ; p<0,00)$. O fator Focalizado no Problema quando comparado com Busca de Suporte Social não apresentou resultados significativos. A estratégia Focalizado no Problema aparece como a mais utilizada $(M=3,36, D P=0,51)$, junto da Busca de Suporte Social $(M=3,30, D P=0,64)$, seguida de Prática Religiosa/Pensamento Fantasioso $(\mathrm{M}=3,12, \mathrm{DP}=0,79)$ e por último a Focalizado na Emoção $(\mathrm{M}=2,78$, $\mathrm{DP}=0,52$ ).

Nas situações "Pedir a um colega que desligue seu celular na aula", "Apresentar trabalho em aula" e "Pedir aos colegas que evitem de lanchar", as estratégias Focalizado no Problema e a Busca de Suporte Social foram as mais utilizadas pelos alunos. Em seguida, para as três situações, aparece a Prática Religiosa/Pensamento Fantasioso e por último, a estratégia Focalizado na Emoção. Para a primeira situação ( $\mathrm{M}=3,43$; $\mathrm{DP}=0,48),(\mathrm{M}=3,29$; $\mathrm{DP}=0,68$ ), $(\mathrm{M}=3,07 ; \mathrm{DP}=0,79),(\mathrm{M}=2,73 ; \mathrm{DP}=0,52)$; para a segunda $(M=3,44 ; D P=0,47),(M=3,29 ; D P=0,66)$, $(\mathrm{M}=2,94 ; \mathrm{DP}=0,83),(\mathrm{M}=2,67 ; \mathrm{DP}=0,48)$ e para $\mathrm{a}$ terceira $(\mathrm{M}=3,33$; $\mathrm{DP}=0,55),(\mathrm{M}=3,31 ; \mathrm{DP}=0,67)$, $(\mathrm{M}=3,13 ; \mathrm{DP}=0,82),(\mathrm{M}=2,89 ; \mathrm{DP}=0,52)$. Foi encontrada diferença significativa entre todas as estratégias com exceção de Focalizado no Problema e Busca de
Suporte Social. Para a primeira situação os seguintes resultados das comparações foram encontrados: Problema/Emoção $(t=8,91 ; p<0,00)$; Problema/Fantasioso ( $t=3,81 ; p<0,00)$; Emoção/Fantasioso $(t=-3,98$; $p<0,00)$; Emoção/Suporte Social $(t=-6,26 ; p<0,00)$; Fantasioso/Suporte Social $(t=-2,05 ; p<0,04)$. Para a segunda verifica-se que para Problema/Emoção $(t=10,02 ; p<0,00)$; Problema/Fantasioso $(t=4,70$; $p<0,00)$; Emoção/Fantasioso $(t=-3,16 ; p<0,00)$; Emoção/Suporte Social $(t=-6,99 ; p<0,00)$; Fantasioso/Suporte Social $(t=-3,13 ; p<0,00)$. Para a terceira situação identificou-se que para Problema/ Emoção $(t=4,94 ; p<0,00)$; Problema/Fantasioso $(t=2,15 ; p<0,03)$; Emoção/Fantasioso $(t=-2,56$; $p<0,01)$; Emoção/Suporte Social $(t=-5,35 ; p<0,00)$; Fantasioso/Suporte Social $(t=-1,93 ; p<0,04)$.

Para a situação "Mobilizar colegas para reivindicar direitos", embora a ordem das estratégias mais utilizadas se mantenha, há diferença significativa entre todas com os seguintes escores $(\mathrm{M}=3,40$; $\mathrm{DP}=0,50)$, $(\mathrm{M}=3,27 ; \mathrm{DP}=0,49),(\mathrm{M}=3,04 ; \mathrm{DP}=0,72),(\mathrm{M}=2,69$; $\mathrm{DP}=0,55)$. Para as comparações foi encontrado: Problema/Emoção $(t=8,56 ; p<0,00)$; Problema/Fantasioso $(t=4,20 ; p<0,00)$; Problema/Suporte Social $(t=2,05 ; p<0,04)$; Emoção/Fantasioso $(t=-4,24$; $p<0,00)$; Emoção/Suporte Social $(t=-7,69 ; p<0,00)$; Fantasioso/Suporte Social $(t=-2,57 ; p<0,01)$.

Na situação "Receber críticas do professor" nota-se que a ordem das estratégias se diferencia das demais, a Prática Religiosa/Pensamento Fantasioso aparece em primeiro lugar $(\mathrm{M}=3,43 ; \mathrm{DP}=0,72)$, seguida da Busca de Suporte Social $(M=3,36 ; D P=0,68)$, Focalizado no Problema $(\mathrm{M}=3,19$; $\mathrm{DP}=0,51)$ e por último Focalizado na Emoção ( $M=2,94 ; D P=0,48$ ). Todos apresentando diferenças significativas. Para as comparações foi encontrado: Problema/Emoção $(t=3,08 ; p<0,00)$; Problema/Fantasioso $(t=-2,69 ; p<0,01)$; Problema/ Suporte Social $(t=-2,26 ; p<0,03)$; Emoção/Fantasioso $(t=-6,29 ; p<0,00)$; Emoção/Suporte Social $(t=-4,87$; $p<0,00)$; Fantasioso/Suporte Social $(t=0,78 ; p<0,44)$.

Os resultados da RLM (Tabela) mostram haver uma predição das variáveis independentes na variável dependente $[\mathrm{F}(2,396)=18,948, p<0,01]$, explicando $8 \%$ dos escores de adaptação acadêmica $\left(R^{2}\right)$. A EMEP e o IHS impactaram significativamente nos escores de adaptação acadêmica, sendo que o maior impacto foi das estratégias de enfrentamento (Beta $=0,245$ ). Os resultados da RLM mostram haver uma predição dos fatores da EMEP nos escores gerais da adapta- 
Tabela

Impacto dos escores totais das estratégias de enfrentamento e habilidades sociais na adaptação acadêmica.

\begin{tabular}{|c|c|c|c|c|c|c|}
\hline \multirow[t]{2}{*}{ QVA-r_Total } & \multicolumn{2}{|c|}{$\begin{array}{l}\text { Coeficientes não } \\
\text { padronizados }\end{array}$} & \multirow{2}{*}{$\begin{array}{c}\begin{array}{c}\text { Coeficientes } \\
\text { padronizados }\end{array} \\
\text { Beta } \\
\end{array}$} & \multirow[t]{2}{*}{$t$} & \multirow[t]{2}{*}{ Sig. } & \multirow[t]{2}{*}{$R^{2}$} \\
\hline & B & Erro-padrão & & & & \\
\hline Constante & 2,147 & 0,193 & - & 11,112 & 0,000 & \\
\hline IHS_Total & 0,132 & 0,048 & 0,132 & 2,720 & 0,007 & 0,080 \\
\hline EMEP_Total & 0,266 & 0,053 & 0,245 & 5,044 & 0,000 & \\
\hline Constante & 1,928 & 0,181 & - & 10,646 & 0,000 & 0,161 \\
\hline EMEP_Problema & 0,307 & 0,041 & 0,365 & 7,576 & 0,000 & - \\
\hline EMEP_Emoção & 0,075 & 0,041 & 0,091 & 1,818 & 0,070 & - \\
\hline $\begin{array}{l}\text { EMEP_Problema/ } \\
\text { Fantasioso }\end{array}$ & $-0,025$ & 0,027 & $-0,046$ & $-0,913$ & 0,362 & - \\
\hline EMEP_Suporte Social & 0,073 & 0,027 & 0,130 & 2,745 & 0,006 & - \\
\hline Constante & 2,875 & 0,148 & - & 19,449 & 0,000 & 0,056 \\
\hline IHS_Enfrentamento & $-0,002$ & 0,037 & $-0,004$ & $-0,065$ & 0,948 & - \\
\hline IHS_Sentimento & 0,160 & 0,041 & 0,219 & 3,926 & 0,000 & - \\
\hline IHS_Conversação & 0,021 & 0,036 & 0,034 & 0,584 & 0,560 & - \\
\hline IHS_Autoexposição & 0,028 & 0,029 & 0,055 & 0,943 & 0,346 & - \\
\hline
\end{tabular}

ção acadêmica $[\mathrm{F}(4,395)=20,075, p<0,001]$, explicando $16,1 \%$ dos escores $\left(R^{2}\right)$. Os fatores: Focalizado no Problema e Busca de Suporte Social apresentaram impacto positivo na adaptação acadêmica. A predição mais forte foi referente ao fator Focalizado no Problema (Beta $=0,365)$. Os resultados também mostraram haver uma predição dos fatores do IHS nos escores gerais da adaptação acadêmica $[\mathrm{F}(5,393)=5,695$, $p<0,001$ ], explicando 5,6\% dos escores $\left(R^{2}\right)$. O fator Autoafirmação na Expressão de Sentimento Positivo apresentou impacto significativo nos escores totais do QVA-r.

\section{Discussão}

No que diz respeito aos resultados relativos às estratégias de enfrentamento independente das situações, as estratégias: Focalizado no Problema e Busca de Suporte Social foram as mais utilizadas, seguidas por Prática Religiosa/Pensamento Fantasioso e Focalizado na Emoção. O mesmo ocorreu nas situações: "solicitar que um colega desligue seu celular em sala", "evite lanchar em aula", bem como, "falar em público" ou "mobilizar colegas". Ao utilizarem a estratégia Focalizado no Problema, os estudantes necessitam ter uma percepção real da situação, mesmo que esta seja desfavorável para ele, e maior concentração para enfrentar e resolver problemas ou situações de estresse (Santeiro et al., 2016). Para Lazarus (1993), a estratégia Focalizado no Problema pode envolver negociação para solução de uma situação de estresse interpessoal, como também a Busca de Suporte de outras pessoas para auxílio na resolução. Segundo Bandeira, Quaglia, Bachetti, Ferreira e Souza (2005), essa Busca de Suporte Social se justifica por envolver situações sociais que requerem expressar insatisfação e/ou solicitar mudança de comportamento, consideradas mais desagradáveis ou incômodas para os estudantes universitários. O fato das duas estratégias, Focalizado no Problema e Busca de Suporte Social, aparecerem juntas nas três primeiras situações, pode demonstrar a tendência dos alunos não só de reduzir uma tensão frente a uma situação estressante, mas também de utilizar estratégias adaptativas para cada situação em particular (Clinciu, 2012). Esses resultados corroboram o estudo de Sicre e Casaro (2014) com 140 estudantes de Psicologia na Argentina que encontraram as estratégias de enfrentamento Focalizado no Problema e Busca de Suporte Social como as mais utilizadas pelos universitários.

Tanto de forma independente quanto para as situações específicas, a estratégia Focalizado na Emoção aparece por último. A esse respeito pode-se 
dizer que provavelmente os alunos não priorizam reações emocionais negativas optando por uma posição mais positiva com a intenção de solucionar as questões estressoras focando no problema. Para Santeiro et al. (2016), a estratégia Focalizado na Emoção acaba dificultando a solução dos problemas de forma lógica e racional, bem como afetando os relacionamentos interpessoais, corroborando os estudos de Carlotto et al. (2015), que encontraram que essa estratégia se correlaciona negativamente com a adaptação acadêmica.

Para a situação "Receber críticas do professor", a estratégia de enfrentamento Prática Religiosa/Pensamento Fantasioso foi a mais utilizada. Essa opção remete a uma espera por soluções que independem da ação do indivíduo (Seidl et al., 2001). Nesse contexto, o estudante adota uma postura passiva e prioriza recorrer a ajuda “divina”. Lidar com críticas não é uma situação confortável, podendo levar o estudante a apresentar comportamentos de raiva, por considerar muito difícil aceitar que outras pessoas apontem erros e apresentem suas dificuldades. Tal situação requer do estudante superar as falhas, reconhecendo insucessos na condução de sua prática acadêmica (Soares, \& Del Prette, 2013). As universidades apontam uma preocupação constante em relação ao número de estudantes que apresentam insucesso acadêmico, caracterizado por baixo rendimento, história acadêmica anterior, pouco envolvimento nas pesquisas feitas pelo professor e conteúdos em dia (Almeida et al., 2012; Manee et al., 2015; Santos et al., 2015; Igue et al., 2008). Portanto, a reciprocidade do professor para com o aluno, utilizando-se de críticas construtivas, pode levar o estudante a envolver-se com mais engajamento no processo acadêmico, manifestando mudança de comportamento, maior consciência de seu papel enquanto universitário e viabilizando um estudo mais eficiente (Carelli, \& Santos, 1998).

Das cinco situações propostas, quatro envolvem enfrentamento e autoafirmação com risco e autoexposição. Demandam reações intensas dos estudantes, pois estão expondo-se a situações de risco frente a comportamentos considerados intrusivos por colegas de turma (Soares, \& Del Prette, 2013). Segundo as autoras, estas situações requerem, do universitário, habilidades para apresentar suas ideias ficando exposto a uma reação desagradável do outro. Em todos os casos identifica-se a necessidade de um repertório amplo de habilidades assertivas para minimizar os riscos e aumentar a possibilidade de sucesso no enfrentamento da situação. As habilidades assertivas estão presentes em situações embaraçosas, que exigem destreza social, habilidade verbal, expressando o que o indivíduo pensa, mesmo em situações complexas. Expressar ideias, mesmo que essas não coadunem com o grupo, significa que é preciso pensar assertivamente, exercendo direitos e deveres. Acredita-se que todos podem manifestar o que pensam, usando a assertividade, exercendo direitos de falar e o dever de entender o que pensam os outros (Soares, \& Del Prette, 2013).

Quanto a hipótese preditiva, se as variáveis habilidades sociais e coping são preditoras da adaptação à universidade, o modelo proposto apontou que estudantes que procuram enfrentar as situações desafiadoras recorrendo a diferentes estratégias (Focalizado no Problema, Busca de Suporte Social) supostamente apresentam melhores condições de lidarem com questões de difícil manejo encontradas no cotidiano universitário. As estratégias são processos cognitivos e comportamentais e que necessariamente não conduzem a respostas mais habilidosas, mas a comportamentos de enfrentamento na busca de resolução da situação adversa. Oliveira et al. (2014) afirmam que no cenário nacional estudos não têm investigado o coping como variável da adaptação acadêmica, apesar de saber que as estratégias de enfrentamento utilizadas pelo estudante podem interferir no sucesso da transição para a universidade. Esses resultados se articulam com outros estudos (Costa, \& Leal, 2006; Crockett et al., 2007; Sicre, \& Casaro, 2014) que evidenciam que a utilização de estratégias Focalizado no Problema, no Humor e na Busca de Suporte Social estiveram relacionadas a uma melhor adaptação à universidade. Em contraste, comportamentos de evitação se correlacionaram com menor ajustamento acadêmico (Carlotto et al., 2015). Outros autores apontam que o envolvimento social na universidade promove o progresso acadêmico (Assis et al., 2013; Gerk, \& Cunha, 2006; Webber, Krylow, \& Zhang, 2013). Observa-se também que se o universitário lida efetivamente com as situações mais críticas, pode ter mais chances de se ajustar à universidade e administrar situações menos críticas. Essas situações podem ser menos frequentes, menos estressantes e menos exigentes quanto a um repertório bem elaborado de 
coping, presumivelmente favorecendo o aluno contra o fracasso ou abandono acadêmico.

Especificamente, a estratégia de enfrentamento que mais apresentou impacto em relação à variável adaptação acadêmica foi a Focalizado no Problema. Estudos têm relacionado positivamente estratégias centralizadas nos problemas à adaptação acadêmica (Crockett et al., 2007; Santeiro et al., 2016; Sicre, \& Casaro, 2014), em detrimento de estratégias evitativas. Além disso, o suporte social também é um recurso que se correlacionou positiva e diretamente com a adaptação acadêmica (Costa, \& Leal 2006; Manee et al., 2015).

A habilidade social de Autoafirmação na Expressão de Sentimento Positivo foi a que apresentou predição mais forte dos escores de adaptação acadêmica. Segundo A. Del Prette e Del Prette (2001), este repertório social se refere à expressão de afetos positivos e de autoafirmação da autoestima, evitando ou minimizando, ao máximo, prejuízos sociais. Segundo estudo conduzido por Soares et al. (2009), esta habilidade foi correlacionada positivamente com fatores como: aquisição do conhecimento para o curso, métodos eficientes de estudo, desenvolvimento da carreira, relacionamento com os professores, adaptação ao curso e gestão do tempo. Os autores concluíram que o envolvimento afetivo proporcionado por esta habilidade social é favorável à realização acadêmica.

\section{Considerações finais}

Este estudo teve como objetivo verificar as relações entre habilidades sociais, adaptação à universidade e estratégias de coping em situações interpessoais difíceis, além de investigar se as variáveis habilidades sociais e coping são preditoras da adaptação à universidade. Os resultados apresentados contribuem para ampliar o entendimento e o uso dos processos cognitivos e comportamentais associados à adaptação ao Ensino Superior, o que possibilita a criação de recursos por parte das universidades para ajudar os estudantes nesse processo. Foi possível repertoriar situações específicas consideradas difíceis no cotidiano do estudante, as quais podem interferir no processo de ajustamento e identificar as estratégias de enfrentamento mais utilizadas pelos alunos que podem promover o desenvolvimento de comportamentos habilidosos que minimizem conflitos sociais.

De forma geral, os resultados confirmam os apontamentos encontrados na literatura. As variáveis coping, habilidades sociais e situações interpessoais merecem atenção em futuros estudos relacionados à adaptação à universidade, considerando o fato de que a pesquisa apontou para aspectos compreendidos como facilitadores da permanência do aluno na instituição e com a qualidade no processo de aprendizagem. Como limitações da pesquisa podemos apresentar o fato de o estudo ter sido realizado numa única cidade, a prevalência de alunos de instituições privadas, bem como a prevalência de alunos do curso de Psicologia. Ademais, compreendendo que a adaptação acadêmica é um fenômeno multideterminado, outras variáveis podem ser inseridas em pesquisas prospectivas, contribuindo com o alargamento das investigações sobre o tema. Assim, investigar o comportamento destas variáveis em amostras de estudantes de outras regiões do território nacional, de outros cursos, bem como considerar dados sociodemográficos como sexo e idade, muito contribuiria para a elaboração de programas de intervenção mais pontuais e efetivos que objetivem o ajustamento do aluno à universidade.

\section{Referências}

Albanaes, P., Bardagi, M. P., Luca, G. G., \& Girelli, S. (2014). Do trote à mentoria: Levantamento das possibilidades de acolhimento ao estudante universitário. Revista Brasileira de Orientação Profissional, 15(2), 143-152.

Almeida, L. S., Araújo, A. M., \& Martins, C. (2016). Transição e adaptação dos alunos do $1^{\circ}$ ano: Variáveis intervenientes e medidas de atuação. In: L. S. Almeida, \& R. V. Castro (Orgs.), Ser estudante no ensino superior: O caso dos estudantes do $1^{\circ}$ ano (pp. 146-164). Portugal: Universidade do Minho.

Almeida, L. S., Marinho-Araújo, C. M., Amaral, A., \& Dias, D. (2012). Democratização do acesso e do sucesso no ensino superior: Uma reflexão a partir das realidades de Portugal e do Brasil. Avaliação (Campinas), 17(3), 899-920. https://doi.org/10.1590/S1414-40772012000300014 
Almeida, L. S., Soares, A. P., \& Ferreira, J. A. G. (2002). Questionário de vivências acadêmicas (QVA-r): Avaliação do ajustamento dos estudantes universitários. Avaliação Psicológica, 1(2), 81-93.

Assis, C. L., Silva, A. P. F., Lopes, M. S., Silva, P. C. B., \& Santini, T. O. (2013). Sintomas de estresse em concluintes do curso de psicologia de uma faculdade privada do norte do país. Mudanças: Psicologia da Saúde, 21(1), 23-28. https://doi.org/10.15603/2176-1019/mud.v21n1p23-28

Baker, S. R. (2003). A prospective longitudinal investigation of social problem-solving appraisals on adjustment to university, stress, health and academic motivation and performance. Personality and Individual Differences, 35(3), 569-591. https://doi.org/10.1016/S0191-8869(02)00220-9

Bandeira, M., Quaglia, M. A. C., Bachetti, L. D. S., Ferreira, T. L., \& Souza, G. G. D. (2005). Comportamento assertivo e sua relação com ansiedade, locus de controle e auto-estima em estudantes universitários. Estudos de Psicologia, 22(2), 111-121. https:// doi.org/10.1590/S0103-166X2005000200001

Bisinoto, C., Rabelo, M. L., Marinho-Araújo, C., \& Fleith, D. S. (2016). Expectativas acadêmicas dos ingressantes da Universidade de Brasília: Indicadores para uma política de acolhimento. In: L. S. Almeida, \& R. V. Castro (Orgs.), Ser estudante no ensino superior: O caso dos estudantes do $1^{\circ}$ ano (pp. 15-31). Portugal: Universidade do Minho.

Carelli, M. J. G., \& Santos, A. D. (1998). Condições temporais e pessoais de estudo em universitários. Psicologia Escolar e Educacional, 2(3), 265-278. https://doi.org/10.1590/S1413-85571998000300006

Carlotto, R. C., Teixeira, M. A. P., \& Dias, A. C. G. (2015). Adaptação acadêmica e coping em estudantes universitários. Psico USF, 20(3), 421-432. https://doi.org/10.1590/1413-82712015200305

Clinciu, A. I. (2012). Adaptation and stress for the first year university students. Procedia: Social and Behavioral Sciences, 78, 718-722. https://doi.org/10.1016/j.sbspro.2013.04.382

Costa, E. S., \& Leal, I. P. (2006). Estratégias de coping em estudantes do ensino superior. Análise Psicológica, 24(2), 189-199.

Crockett, L. J., Iturbide, M. I., Torres Stone, R. A., McGinley, M., Raffaelli, M., \& Carlo, G. (2007). Acculturative stress, social support, and coping: Relations to psychological adjustment among mexican american college students. Cultural Diversity and Ethnic Minority Psychology, 13(4), 347. https:/ / doi.org/10.1037/ 1099-9809.13.4.347

Del Prette, A., \& Del Prette, Z. A. P. (2001). Psicologia das relações interpessoais: Vivências para o trabalho em grupo. Petrópolis, RJ: Vozes.

Del Prette, A., \& Del Prette, Z. A. P. (2003). Assertividade, sistema de crenças e identidade social. Psicologia em Revista, 9(1), 125-136.

Del Prette, Z. A. P., \& Del Prette, A. (2001). Inventário de habilidades sociais (IHS-Del-Prette): Manual de aplicação, apuração e interpretação. São Paulo, SP: Casa do Psicólogo.

Del Prette, Z. A. P., \& Del Prette, A. (2010). Habilidades sociais e análise do comportamento: Proximidade histórica e atualidades. Revista Perspectiva, 1(2), 104-115.

Fagundes, C.V. (2012). Transição ensino médio-educação superior: Qualidade no processo educativo. Revista Educação por Escrito, 3(1), 62-73.

Feldt, R. C., Graham, M., \& Dew, D. (2011). Measuring adjustment to college: Construct validity of the student adaptation to college questionnaire. Measurement and Evaluation in Counseling and Development, 44(2), 92-104.

Gerk, E., \& Cunha, S. M. (2006). As habilidades sociais na adaptação de estudantes ao ensino superior. In M. Bandeira, Z. A. P. Del Prette, \& A. Del Prette (Orgs.), Estudos sobre habilidades sociais e relacionamento interpessoal (pp. 129-146). São Paulo, SP: Casa do Psicólogo.

González-Torres, M. C., \& Artuch-Garde, R. (2014). Resilience and coping strategy profiles at university: Contextual and demographic variables. Eletronic Journal of Research in Educational Psychology, 12(3), 1696-2095. https://doi.org/10.14204/ejrep.34.14032

Granado, J. I. F., Santos, A. A., Almeida, L. S., Soares, A. P., \& Guisande, M. A. (2005). Integração acadêmica de estudantes universitários: Contributos para a adaptação e validação do QVA-r no Brasil. Psicologia e Educação, 4(1), 33-43. 
Igue, E. A., Bariani, I. C. D., \& Milanesi, P.V. B. (2008). Vivência acadêmica e expectativas de universitários ingressantes e concluintes. PsicoUSF, 13(2), 155-164. https://doi.org/10.1590/S1413-82712008000200003

Iksan, Z. H., Zakaria, E., Meerah, T. S. M., Osman, K., Lian, D. K. C., Mahmud, S. N. D. et al. (2012). Communication skills among university students. Procedia-Social and Behavioral Sciences, 59, 71-76.

Kuncharin, L., \& Mohamad, A. R. B. (2014). Coping strategies on academic performance among undergraduate students in Thailand. The Standard International Journals Transactions on Industrial, Financial \& Business Management, 2(3), 58-61.

Lazarus, R. S. (1993). From psychological stress to the emotions: A history of changing outlooks. Annual Review of Psychology, 44, 1-21. https://doi.org/10.1146/annurev.ps.44.020193.000245

Lazarus, R. S., \& Folkman, S. (1984). Stress, appraisal, and coping. New York, NY: Springer.

Manee, F. M., Khoiee, S. A., \& Eghbal, M. K. (2015). Comparison of the efficiency of self-awareness, stress management, effective communication life skill trainings on the social and academic adjustment of first-year students. Research in Psychology and Behavioral Sciences, 3(2), 18-24. https://doi.org/10.1146/10.12691/rpbs-3-2-1

Mofidi, T., El-Alayli, A., \& Brown, A. A. (2014). Trait gratitude and grateful coping as they relate to college student persistence, success, and integration in school. Journal of College Student Retention, 16(3) 325-349. https://doi.org/10.2190/CS.16.3.b

O’Brien, L., Mathieson, K., Leafman, J., \& Rice-Spearman, L. (2012). Level of stress and common coping strategies among physician assistant students. The Journal of Physician Assistant Education, 23(4), 25-29.

Oliveira, C. T., Carlotto, R. C., Vasconcellos, S. J. L., \& Dias, A. C. G. (2014). Adaptação acadêmica e coping em estudantes universitários brasileiros: Uma revisão de literatura. Revista Brasileira de Orientação Profissional, 15(2), 177-186.

Pasquali, L. (2015). Delineamento de pesquisa em ciência. São Paulo, SP: Vetor.

Santeiro, T. V., Yoshida, E. M. P. Peixoto, E. M., Rocha, G. M. A., \& Zanini, D. S. (2016). Diferenças conceituais e empíricas entre eficácia adaptativa e coping. Estudos Interdisciplinares em Psicologia, 7(1), 2-19. https:// doi.org/10.5433/2236-6407.2016v7n1p02

Santos, A. S., Oliveira, C. T., \& Dias, A. N. G. (2015). Características das relações dos universitários e seus pares: Implicações na adaptação acadêmica. Revista Psicologia: Teoria e Prática, 17(1), 150-163. https:// doi.org/10.15348/1980-6906/psicologia.v17nlp150-163.

Seidl, E. M. F., Tróccoli, B. T., \&Zannon, C. M. L. D. C. (2001). Análise fatorial de uma medida de estratégias de enfrentamento. Psicologia: Teoria e Pesquisa, 17(3), 225-234. https:// doi.org/10.1590/S0102-37722001000300004

Sicre, E., \& Casaro, L. (2014). Estrategias de afrontamiento en estudiantes de psicología. Revista de Psicología, 10(20), 61-72.

Soares, A. B., \& Del Prette, Z. A. P. (2013). Guia teórico-prático para superar as dificuldades interpessoais na universidade. Rio de Janeiro, RJ: Appris.

Soares, A. B., Francischetto, V., Peçanha, A. P. C. L., Miranda, J. M., \& Dutra, B. M. S. (2013). Inteligência e competência social na adaptação à universidade. Estudos de Psicologia, 30(3), 317-328.

Soares, A. B., Gomes, G., Maia, F. A., Gomes, C. A., \& Monteiro, M. C. (2016b). Relações interpessoais na universidade: O que pensam estudantes da graduação em psicologia? Estudos Interdisciplinares em Psicologia, 7(1), 56-76. https://doi.org/10.5433/2236-6407.2016v7nlp56

Soares, A. B., Mello, T. V. S., \& Baldez, M. O. (2011). Vivências acadêmicas em estudantes universitários do estado do Rio de Janeiro. Interação em Psicologia, 15(1), 59-69. https://doi.org/10.5380/psi.v15i1.16049

Soares, A. B., Monteiro, M. C., Maia, F. A., Buscacio, R. C. Z., Rodrigues, P. V. S., Medeiros, H. C. P. et al. (2016). Revisão sistemática da bibliografia sobre vivências acadêmicas em estudantes universitários no Brasil. Psicologia, Educação e Cultura, 20(1), 119-137.

Soares, A. B., Mourão, L., Santos, A. A. A., \& Mello, T. V. (2015). Habilidades sociais e vivência acadêmica de estudantes universitários. Interação em Psicologia, 19(2), 211-223. https://doi.org/10.5380/psi.v19i2.31663

Soares, A. B., Poubel, L. N., \& Mello, T. V. S. (2009). Habilidades sociais e adaptação acadêmica: Um estudo comparativo em instituições de ensino público e privado. Aletheia, (29), 27-42. 
Soares, A. B., Seabra, A. M. R., \& Gomes, G. (2016a). Vivências acadêmicas e habilidades sociais: Um estudo correlacional com universitários do primeiro ano de instituições do Rio de Janeiro. In: A. B. Soares, L. Mourão, M. M. P. E. Mota (Orgs), Estudante universitário brasileiro: Características cognitivas, habilidades relacionais e transição para o mercado de trabalho (pp. 143-156). Curitiba, PR: Appris.

Vitaliano, P. P., Russo, J., Carr, J. E., Maiuro, R. D. \& Becker, J. (1985). The ways of coping checklist: Revision and psychometric properties. Multivariate Behavioral Research, 20(1), 3-26.

Webber, K. L., Krylow, R. B., \& Zhang, Q. (2013). Does involvement really matter? Indicators of college student success and satisfaction. Journal of College Student Development, 54(6), 591-611. https://doi.org/10.1353/ csd.2013.0090

\section{Adriana Benevides Soares}

Doutora. Professora da Universidade Salgado de Oliveira e da Universidade do Estado do Rio de Janeiro. Rio de Janeiro - RJ. Brasil.

E-mail: adribenevides@gmail.com

D http://orcid.org/0000-0001-8057-6824

\section{Marcia Cristina Monteiro}

Doutora pela Universidade Salgado de Oliveira. São Paulo - SP. Brasil.

E-mail: marcia_lauria@yahoo.com.br

http://orcid.org/0000-0003-3455-2690

Marisangela Siqueira de Souza

Mestre pela Universidade Salgado de Oliveira. São Paulo - SP. Brasil.

E-mail: profmarisangela@gmail.com

D https://orcid.org/0000-0002-9366-7706

\section{Fátima Almeida Maia}

Doutora pela Universidade Salgado de Oliveira. São Paulo - SP. Brasil.

E-mail: maiafalmeida@gmail.com

D http://orcid.org/0000-0002-7035-3405

Humberto Cláudio Passeri Medeiros

Programa de Pós-Graduação stricto sensu em Psicologia. Mestrado em Psicologia Área de Concentração: Psicologia Social. Mestre.

E-mail: hcpasseri@gmail.com

D https://orcid.org/0000-0002-1091-8136

Roberta de Souza Nogueira Barros

Mestre pela Universidade Salgado de Oliveira. São Paulo - SP. Brasil.

E-mail: rnbarros@yahoo.com.br

https://orcid.org/0000-0003-3240-1960

Recebido 10/08/2017

Aceito 03/07/2018

Received 08/10/2017

Approved 07/03/2018 
Recibido 10/08/2017

Aceptado 03/07/2018

Como citar: Soares, A. B.; Monteiro, M. C.; Souza, M. S.; Maia, F. A.; Medeiros, H. C. P. \& Barros, R. S. N. (2019). Situações Interpessoais Difíceis: Relações entre Habilidades Sociais e Coping na Adaptação Acadêmica. Psicologia: Ciência e Profissão, 39, 1-13. https://doi.org/10.1590/1982-3703003183912

How to cite: Soares, A. B.; Monteiro, M. C.; Souza, M. S.; Maia, F. A.; Medeiros, H. C. P. \& Barros, R. S. N. (2019). Difficult Interpersonal Situations: Relations between Social Skills and Coping in Academic Adaptation. Psicologia: Ciência e Profissão, 39, 1-13. https://doi.org/10.1590/1982-3703003183912

Cómo citar: Soares, A. B.; Monteiro, M. C.; Souza, M. S.; Maia, F. A.; Medeiros, H. C. P. \& Barros, R. S. N. (2019). Situaciones Interpersonales Difíciles: Relaciones entre Habilidades Sociales y Coping en la Adaptación Académica. Psicologia: Ciência e Profissão, 39, 1-13. https://doi.org/10.1590/1982-3703003183912 